\title{
Neural-Network Methods for Boundary Value Problems with Irregular Boundaries
}

\author{
Isaac Elias Lagaris, Aristidis C. Likas, Member, IEEE, and Dimitrios G. Papageorgiou
}

\begin{abstract}
Partial differential equations (PDEs) with boundary conditions (Dirichlet or Neumann) defined on boundaries with simple geometry have been successfully treated using sigmoidal multilayer perceptrons in previous works. This article deals with the case of complex boundary geometry, where the boundary is determined by a number of points that belong to it and are closely located, so as to offer a reasonable representation. Two networks are employed: a multilayer perceptron and a radial basis function network. The later is used to account for the exact satisfaction of the boundary conditions. The method has been successfully tested on two-dimensional and three-dimensional PDEs and has yielded accurate results.
\end{abstract}

Index Terms-Boundary value problems, engineering problems, irregular boundaries, neural networks, partial differential equations (PDEs), penalty method, multilayer perceptron, radial basis function (RBF) networks.

\section{INTRODUCTION}

$\mathbf{N}$ EURAL networks have been employed before to solve boundary and initial value problems [1] as well as eigenvalue problems [2]. The cases treated in the above mentioned articles were for simple orthogonal box boundaries either finite or extended to infinity. However, when one deals with realistic problems, such as the human head-neck system [3] or the flow and mass transfer in chemical vapor deposition reactors [4], the boundary is highly irregular and cannot be described in terms of simple geometrical shapes, that in turn would have allowed for a relatively simple modeling scheme.

In this article we propose a method capable of dealing with such kind of irregular boundaries (with either Dirichlet or Neumann boundary conditions). As before [1], [2], our approach is based on the use of feedforward artificial neural networks (ANNs) whose approximation capabilities have been widely acknowledged [7], [8]. More specifically, the proposed approach is based on the synergy of two feedforward ANNs of different types: a multilayer perceptron (MLP) as the basic approximation element and a radial basis function (RBF) network used to satisfy the boundary conditions (BCs). In addition, our approach relies on the availability of efficient multidimensional optimization software [5], that is used for the neural network training.

The solution of differential equations in terms of ANNs possesses several attractive features:

Manuscript received January 29, 1999; revised December 3, 1999 and June $5,2000$.

I. E. Lagaris and A. C. Likas are with the Department of Computer Science, University of Ioannina, 45110 Ioannina, Greece (e-mail: lagaris@cs.uoi.gr; arly@cs.uoi.gr).

D. G. Papageorgiou is with the Network Operations Center, University of Ioannina, 45110 Ioannina, Greece (e-mail: jimmy@ noc.uoi.gr).

Publisher Item Identifier S 1045-9227(00)07865-6.
1) infinitely differentiable closed analytic form;

2) superior interpolation properties as compared to well-established methods such as finite elements [1], [2];

3) small number of parameters;

4) suitability for efficient implementation on parallel computers;

5) implementability on existing specialized hardware (neuroprocessors) a fact that will result to a significant computational speedup.

In the next section we describe the proposed method and derive useful formulas, while in Section III, we discuss implementation procedures and numerical techniques. In Section IV we illustrate the method by means of examples and compare our results to analytically known ones. Finally Section V contains conclusions and directions for future research.

\section{DESCRIPTION OF THE METHOD}

We will examine PDEs of the form

$$
L \Psi=f
$$

where $L$ is a differential operator and $\Psi=\Psi(\vec{x})(\vec{x} \in D \subset$ $\left.R^{(n)}\right)$ with Dirichlet or Neumann BCs. The boundary $B=\partial D$ can be any arbitrarily complex geometrical shape. We consider that the boundary is defined as a set of points that are chosen so as to represent its shape with reasonable accuracy. Suppose that $M$ points $\vec{r}_{1}, \vec{r}_{2}, \ldots, \vec{r}_{M} \in B$ are chosen to represent the boundary and hence the boundary conditions are given by

$$
\Psi\left(\vec{r}_{i}\right)=b_{i}, \quad i=1,2, \ldots, M \quad \text { (Dirichlet) }
$$

or

$$
\vec{n}_{i} \cdot \nabla \Psi\left(\vec{r}_{i}\right)=c_{i}, \quad i=1,2, \ldots, M \quad \text { (Neumann) }
$$

where $\vec{n}_{i}$ is the outward unit vector, normal to the boundary at the point $\vec{r}_{i}$.

To obtain a solution to the above differential equation, the $\mathrm{col}$ location method [9] is adopted which assumes the discretization of the domain $D$ into a set of points $\hat{D}$ (these points are denoted by $\left.\vec{x}_{i}, i=1, \ldots, K\right)$. The problem is then transformed into the following system of equations:

$$
\begin{aligned}
L \Psi\left(\vec{x}_{i}\right) & =f\left(\vec{x}_{i}\right), \quad \forall \vec{x}_{i} \in \hat{D}, \quad \text { with } \\
\Psi\left(\vec{r}_{i}\right) & =b_{i}, \quad \text { or } \quad \vec{n}_{i} \cdot \nabla \Psi\left(\vec{r}_{i}\right)=c_{i}, \quad \forall \vec{r}_{i} \in B .
\end{aligned}
$$

Let $\Psi_{M}(\vec{x}, p)$ denote a trial solution to the above problem where $p$ stands for a set of model parameters to be adjusted. 
In this way, the problem is transformed into the following constrained minimization problem:

$$
\min _{p} E(p)=\sum_{i=1}^{K}\left(L \Psi_{M}\left(\vec{x}_{i}, p\right)-f\left(\vec{x}_{i}\right)\right)^{2}
$$

subject to the constraints imposed by the BCs

$$
\Psi_{M}\left(\vec{r}_{i}, p\right)=b_{i}, \quad i=1,2, \ldots, M \quad \text { (Dirichlet) }
$$

or

$$
\vec{n}_{i} \cdot \nabla \Psi_{M}\left(\vec{r}_{i}, p\right)=c_{i}, \quad i=1,2, \ldots, M \quad \text { (Neumann). }
$$

The constrained optimization problem above may be tackled in a number of ways.

1) Devise a model $\Psi_{M}(\vec{x}, p)$, such that the constraints are exactly satisfied by construction and hence use unconstrained optimization techniques.

2) Use a suitable constrained optimization method for nonlinear constraints. For instance: Lagrange multipliers, active set methods, or a penalty function approach [11].

A model suitable for the first approach is based on the synergy of two feedforward neural networks of different type, and it can be written as

$$
\Psi_{M}(\vec{x}, p)=N(\vec{x}, p)+\sum_{l=1}^{M} q_{l} e^{-\lambda\left|\vec{x}-\alpha \vec{r}_{l}+\vec{h}\right|^{2}}
$$

where $|\cdot|$ denotes the Euclidean norm and $N(\vec{x}, p)$ is a multilayer perceptron (MLP) with $p$ denoting the set of its weights and biases. The sum in the above equation represents an RBF network with $M$ hidden units that all share a common exponential factor $\lambda$. Here $\alpha$ is a scalar constant and $\vec{h}$ is a constant vector. The parameters $\lambda, \alpha$ and $\vec{h}$ are chosen appropriately so as to ease the numerical task.

For a given set $p$ of MLP parameters, the coefficients $q_{l}$ are uniquely determined by requiring that the boundary conditions are satisfied, i.e.,

$$
b_{i}-N\left(\vec{r}_{i}, p\right)=\sum_{l=1}^{M} q_{l} e^{-\lambda\left|\vec{r}_{i}-\alpha \vec{r}_{l}+\vec{h}\right|^{2}} \quad \text { (Dirichlet) }
$$

or

$$
\begin{aligned}
c_{i}-\vec{n}_{i} \cdot \nabla N\left(\vec{r}_{i}, p\right)= & -2 \lambda \sum_{l=1}^{M} q_{l} e^{-\lambda \mid \vec{r}_{i}-\alpha \vec{r}_{l}+\vec{h}^{2}} \vec{n}_{i} \\
& \cdot\left(\vec{r}_{i}-\alpha \vec{r}_{l}+\vec{h}\right) \quad \text { (Neumann) }
\end{aligned}
$$

for $i=1,2, \ldots, M$. Therefore, in order to obtain the parameters $q_{i}$ that satisfy the BCs, one has to solve a linear system, which for the Dirichlet case reads

$$
A q=\gamma, \quad A_{i j}=e^{-\lambda\left|\vec{r}_{i}-\alpha \vec{r}_{j}+\vec{h}\right|^{2}}, \quad \gamma_{i}=b_{i}-N\left(\vec{r}_{i}, p\right)
$$

while for the Neumann case

$$
\begin{aligned}
K q & =\delta, \quad K_{i j}=-2 \lambda e^{-\lambda \mid \vec{r}_{i}-\alpha \vec{r}_{j}+\vec{h}^{2}} \vec{n}_{i} \cdot\left(\vec{r}_{i}-\alpha \vec{r}_{j}+h\right), \\
\delta_{i} & =c_{i}-\vec{n}_{i} \cdot \nabla N\left(\vec{r}_{i}, p\right) .
\end{aligned}
$$

Alternatively, one may consider a penalty function method to solve the constrained optimization problem. The model in this case is simply $\Psi_{M}(\vec{x}, p)=N(\vec{x}, p)$. The error function to be minimized is then given by

$$
\begin{aligned}
E(p, \eta)= & \sum_{i=1}^{K}\left(L N\left(\vec{x}_{i}, p\right)-f\left(\vec{x}_{i}\right)\right)^{2} \\
& +\eta \sum_{i=1}^{M}\left(N\left(\vec{r}_{i}, p\right)-b_{i}\right)^{2} \quad \text { (Dirichlet) }
\end{aligned}
$$

or

$$
\begin{aligned}
E(p, \eta)= & \sum_{i=1}^{K}\left(L N\left(\vec{x}_{i}, p\right)-f\left(\vec{x}_{i}\right)\right)^{2} \\
& +\eta \sum_{i=1}^{M}\left(\vec{n}_{i} \cdot \nabla N\left(\vec{r}_{i}, p\right)-c_{i}\right)^{2} \quad \text { (Neumann) }
\end{aligned}
$$

where the penalty factor $\eta$, takes on higher and higher positive values depending on how accurately the BCs are to be satisfied.

The model based on the MLP-RBF synergy satisfies exactly the $\mathrm{BCs}$ but is computationally expensive since at every evaluation of the model one needs to solve a linear system which may be quite large. Moreover, since many efficient optimization methods need the gradient of the objective function, one has to solve an additional linear system of the same order for each gradient component as it will be shown in the next section. On the other hand, the penalty method is very efficient, but does not satisfy exactly the BCs. In practice a combination of these two methods may be used profitably: the penalty method is used to obtain a reasonable model that satisfies the BCs approximately and is then refined using the synergy method for a few iterations. This is done mainly in order to ensure that the $\mathrm{BCs}$ are satisfied exactly. We used the above combination in all of our experiments and our results are quite encouraging.

\section{IMPLEMENTATION AND NUMERICAL TECHNIQUES}

The MLPs we have considered contain one hidden layer with sigmoidal hidden units and a linear output that is computed as

$$
N(\vec{x}, p)=\sum_{i=1}^{H} v_{i} \sigma\left(\sum_{j=1}^{n} w_{i j} x_{j}+u_{i}\right)
$$

where $n$ is the number of input units, $H$ is the number of the hidden units, and $\sigma(z)=\left[1+e^{-z}\right]^{-1}$.

In order to minimize the error $E(p)$, optimization techniques are employed that require the computation of the derivatives $\partial E / \partial p$ and, consequently, the derivatives $\partial \Psi_{M} / \partial p$ which are 
listed as follows:

$$
\frac{\partial \Psi_{M}(\vec{x}, p)}{\partial p}=\frac{\partial N(\vec{x}, p)}{\partial p}+\sum_{l=1}^{M} \frac{\partial q_{l}}{\partial p} e^{-\lambda\left(\vec{x}-\alpha \vec{r}_{l}+\vec{h}\right)^{2}} .
$$

Since for the case of Dirichlet BCs (11)

$$
q_{l}=\sum_{i=1}^{M} A_{l i}^{-1}\left(b_{i}-N\left(\vec{r}_{i}, p\right)\right)
$$

and for the case of Neumann BCs (12)

$$
q_{l}=\sum_{i=1}^{M} K_{l i}^{-1}\left(c_{i}-\vec{n}_{i} \cdot \nabla N\left(\vec{r}_{i}, p\right)\right)
$$

we correspondingly get

$$
\frac{\partial q_{l}}{\partial p}=-\sum_{i=1}^{M} A_{l i}^{-1} \frac{\partial N\left(\vec{r}_{i}, p\right)}{\partial p}
$$

and

$$
\frac{\partial q_{l}}{\partial p}=-\sum_{i=1}^{M} K_{l i}^{-1} \vec{n}_{i} \cdot \nabla \frac{\partial N\left(\vec{r}_{i}, p\right)}{\partial p}
$$

i.e., one has to solve as many $M \times M$ linear systems as the number of the parameters $p$. Derivatives of the MLP with respect to either the parameters $p$ or the input variables $\vec{x}$ can be easily derived and are given in [1], [2].

In order to apply the proposed method, the values of $\lambda, \alpha$ and $\vec{h}$ must be specified. These define the linear systems (matrices $A$ and $K$ ). In our experiments the linear systems were solved using standard $L U$ decomposition. We did not use any special methods for sparse linear systems nor any parallel programming techniques.

For the Dirichlet case $\alpha=1$ and $\vec{h}=0$ were adequate to produce a nonsingular well-behaved matrix $A$. For large values of $\lambda$, the Gaussian terms in the RBF are all highly localized so that they affect the model only in the neighborhood of the boundary points. In other words, the RBF contributes a "correction" that accounts for the BCs. For small values of $\lambda$, the matrix looses rank and becomes singular. So $\lambda$ must be selected with caution. A good choice is found to be: $\lambda \approx 1 / d$, where $d$ is the minimum distance between any two points on the boundary, i.e., $d=\min _{i, j}\left[\left|\vec{r}_{i}-\vec{r}_{j}\right|\right]$, where $i, j=1,2, \ldots, M$. Note that different values $\lambda_{i}$ may also be used instead of a common one. In that case the corresponding $d_{i}$ would be the distance of the closest boundary neighbor to point $\vec{r}_{i}$, i.e., $d_{i}=\min _{j}\left[\left|\vec{r}_{j}-\vec{r}_{i}\right|\right]$, where $i=1,2, \ldots, M$. However, a common $\lambda$ leads to a symmetric matrix $A$ that in turn renders the linear system easier to solve.

For the Neumann case $\lambda$ was chosen again as before, but $\alpha$ had to be different than one and also $\vec{h} \neq 0$ to avoid creating a singular matrix $K$. We took in all cases $\alpha=0.95$ and $\vec{h}$ having all its components equal to 0.1 .

Training of the MLP network so as to minimize the error can be accomplished using any minimization procedure such as gradient descent (backpropagation or any of its variants), conjugate gradient, Newton methods, etc. Many effective minimization techniques are provided by the Merlin/MCL multidimensional optimization system [5], [6] which has been employed in our experiments. From the variety of the minimiza- tion methods offered by the Merlin optimization environment, the quasi-Newton BFGS method [11] seemed to have the best performance [10].

When solving problems requiring several hundreds of boundary points (and thousands of domain points) the method may become relatively slow. There are several techniques that may be applied in order to accelerate the process. The linear systems are sparse and hence one can employ iterative sparse solvers instead of the $L U$ decomposition used here. When computing the gradient of the error function, one has to solve many linear systems with identical left-hand sides and hence one may use special methods that currently are under investigation and development [12]. Parallel programming techniques for machines with many processors are also applicable. The most efficient implementation however would be the one that will utilize specialized hardware (neuroprocessors).

We describe now the strategy followed in detail.

1) Initially the penalty function formulation [(13) or (14)] is used, with $\eta$ starting from 100 and reaching up to 10000 in all tests, to obtain an MLP network that approximates the solution both inside the domain and on the boundary. Since this approach is efficient this task completes rather quickly.

2) The MLP-RBF method is then started, employing the MLP network that was previously obtained by the penalty method. Therefore the MLP-RBF method starts from a low error value and requires only a few optimization steps in order to yield a solution which satisfies the BCs exactly. The RBF network contributes as a correction to the MLP, mainly around the boundary points.

The examples provided in the following section indicate that the above "two-stage" approach is very efficient and provides accurate solutions. Moreover, the reported accuracy can be improved further, by increasing the number of hidden units in the MLP network.

\section{EXAMPLES}

We present three examples in two dimensions and one example in three dimensions. The first two-dimensional (2-D) example is a linear Dirichlet problem also treated in [1] by the method presented there and by the finite-element method (FEM) in order to compare to solutions obtained via an established technique. The boundary is a square and its geometrical simplicity allowed for the scheme used in [1]. Here we pretend that the square is an irregular boundary, so that it is defined by set of points that belong to it, and hence we treat it as such with the method described in the present article, again for purposes of comparison. Since we find that our new method retains the qualities of its ancestor, shown to have certain advantages over the FEM, in the subsequent examples we compare only to the exact, analytically known solutions making no further reference to the FEM. The second example is a highly nonlinear problem and we solve it with Dirichlet BCs considering a star-shaped domain. The third example treats the same PDE with Neumann BCs inside a cardioid domain. Finally in three dimensions we treat again a nonlinear problem in a domain that is one octant of the space between two concentric spherical shells. 


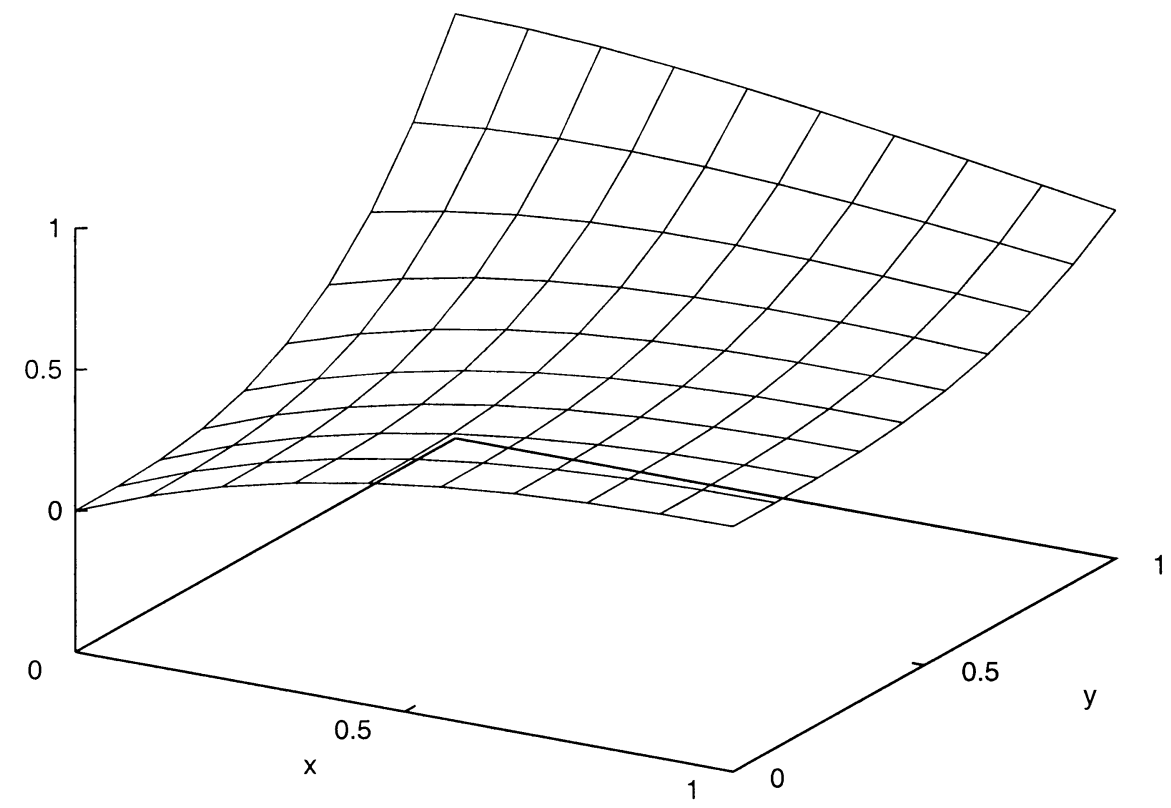

Fig. 1. Exact solution of problem 1.

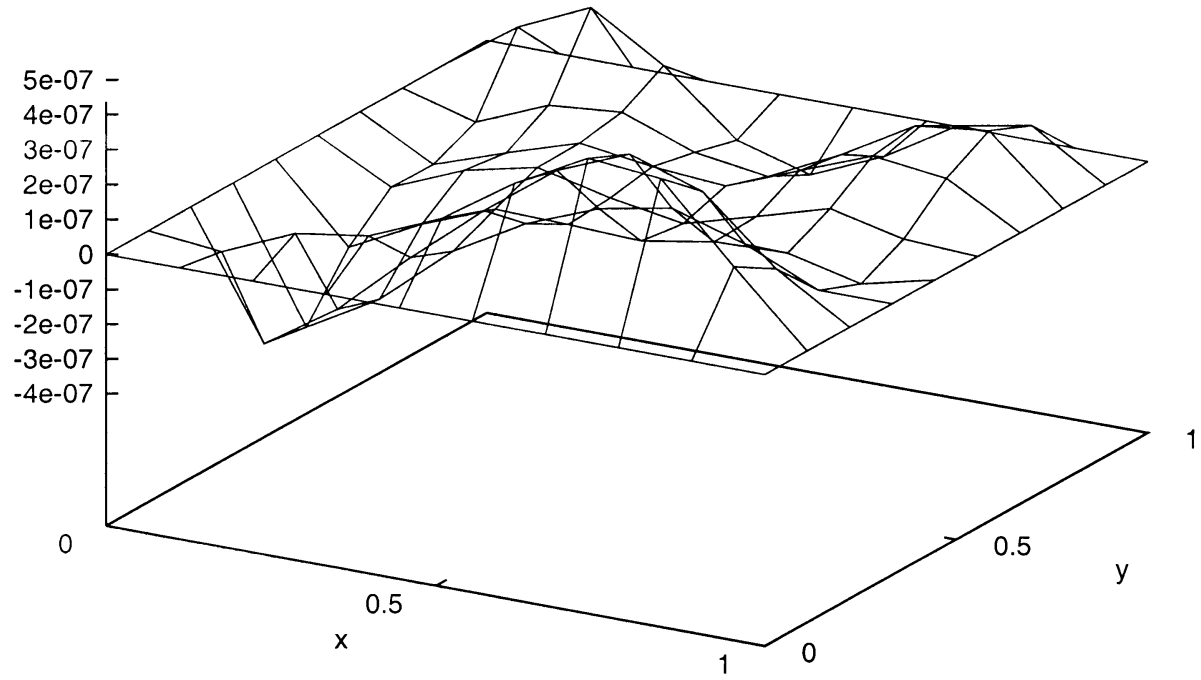

Fig. 2. Accuracy of obtained solution for problem 1 with Dirichlet BCs.

\section{A. 2-D Problems}

Problem 1: Consider the linear problem

$$
\nabla^{2} \Psi(x, y)=e^{-x}\left(x-2+y^{3}+6 y\right), \quad x, y \in[0,1]
$$

with Dirichlet boundary conditions

$$
\begin{aligned}
& \Psi(0, y)=y^{3}, \quad \Psi(1, y)=\frac{1+y^{3}}{e}, \quad \Psi(x, 0)=x e^{-x} \\
& \Psi(x, 1)=e^{-x}(1+x) .
\end{aligned}
$$

The analytic solution is: $\Psi_{a}(x, y)=e^{-x}\left(x+y^{3}\right)$. This example has been treated in [1] by a simpler neural-network model and by the Galerkin FEM. According to the results reported in
[1], the neural-network approach seems to have certain advantages both in efficiency and in, interpolation accuracy.

For comparison purposes, the same problem is treated here by picking points on the square boundary as if it were an irregular shape. More specifically, we consider points $(x, y)$ on the boundary, by dividing the interval $[0,1]$ on the $x$ axis and $y$ axis, respectively, using equidistant points. The total number of points taken on the boundary is $M=36$. Inside the definition domain we pick points on a rectangular grid by subdividing the $[0,1]$ interval in ten equal subintervals that correspond to nine points in each direction. Thus a total of $K=81$ points are selected. The analytic solution is presented in Fig. 1, while the accuracy $\left|\Psi_{M}(x, y)-\Psi_{a}(x, y)\right|$ of the obtained solution using an MLP with 20 hidden units is presented in Fig. 2. Comparing this solution with the one obtained in [1] we can conclude that 


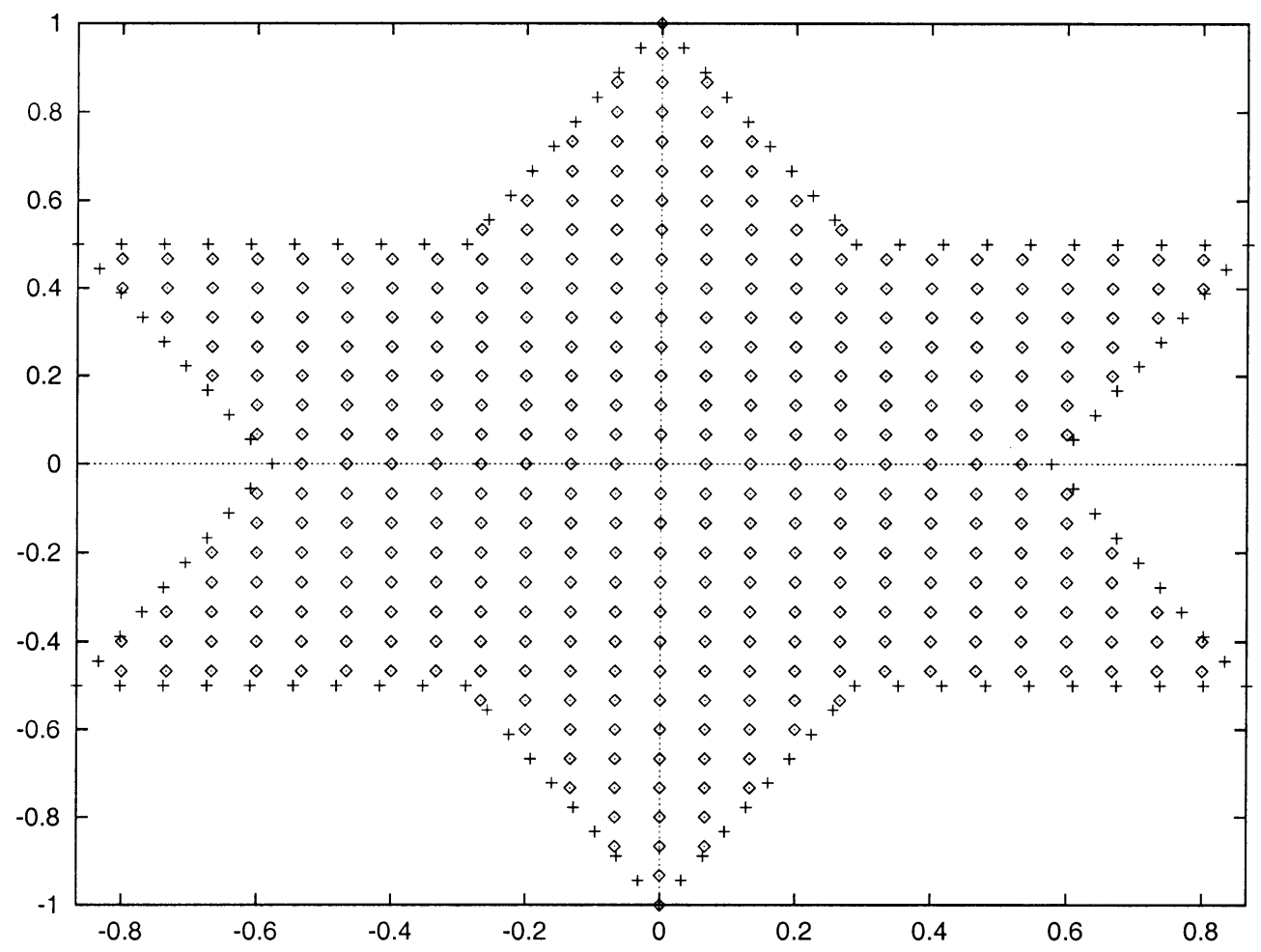

Fig. 3. The star-shaped domain and the boundary points corresponding to problem 2. Boundary points are shown as crosses.

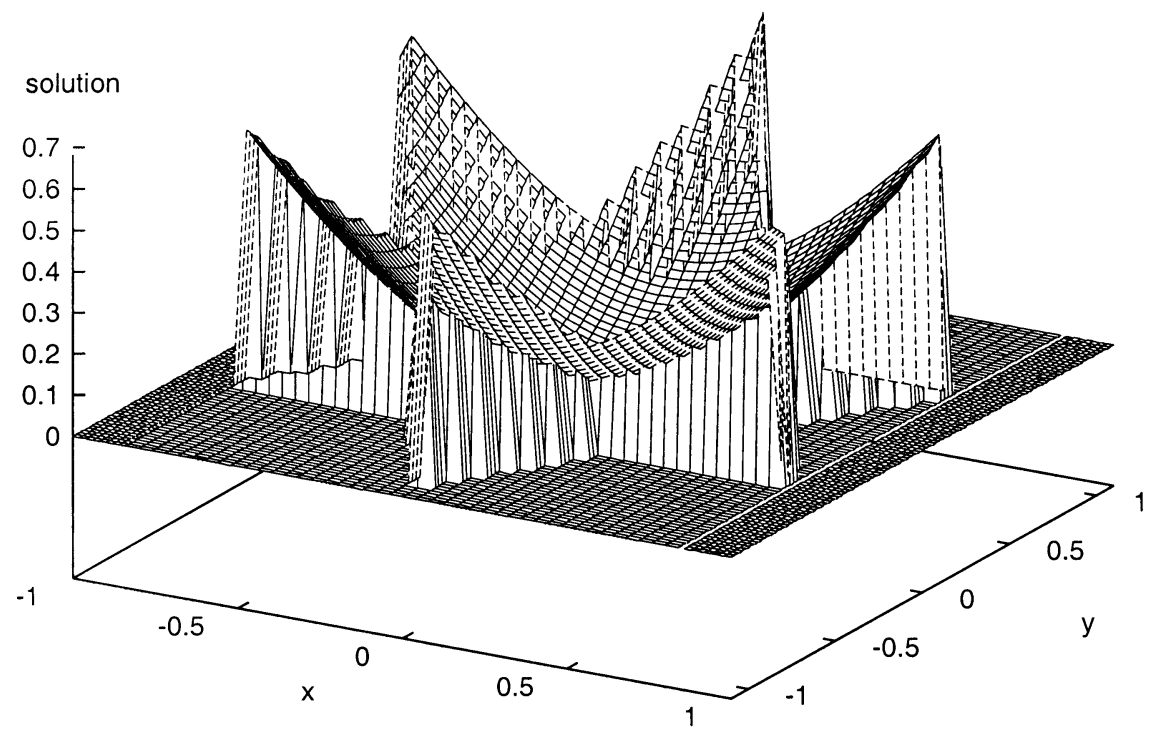

Fig. 4. Exact solution of problem 2 .

the proposed method is equally effective and retains its advantages over the Galerkin FEM as well.

Problem 2: The following highly nonlinear problem is considered:

$$
\nabla^{2} \Psi(x, y)+e^{\Psi(x, y)}=1+x^{2}+y^{2}+\frac{4}{\left(1+x^{2}+y^{2}\right)^{2}}
$$

with the star-shaped domain displayed in Fig. 3. The analytical solution is $\Psi_{a}(x, y)=\log \left(1+x^{2}+y^{2}\right)$ (displayed in Fig. 4) and it has been used to compute the values at the boundary points. We have considered both Dirichlet and Neumann BCs with $M=109$ boundary points and $K=391$ grid points. An MLP with 20 hidden units was used. The accuracy of the obtained solution is displayed in Fig. 5 for the case of Dirichlet BCs, while Fig. 6 displays the output of the RBF network, which contributes as a correction term to satisfy the BCs exactly. Similar results are obtained for the case of Neumann BCs.

To show the interpolative ability of our solutions, the plot in Fig. 5 was made using points belonging to a finer grid (test points), in addition to the collocation (training) points. We found that the accuracy of the solution at these intermediate test points is maintained at the level of the neighboring training ones. This 


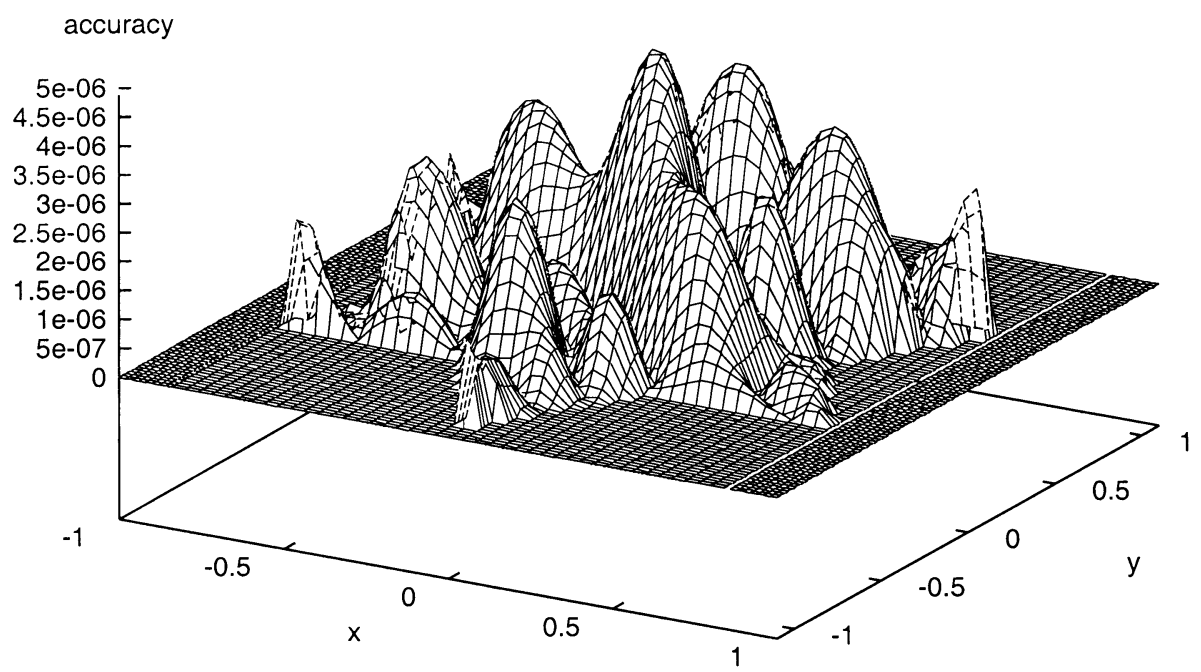

Fig. 5. Accuracy of obtained solution for problem 2 with Dirichlet BCs.

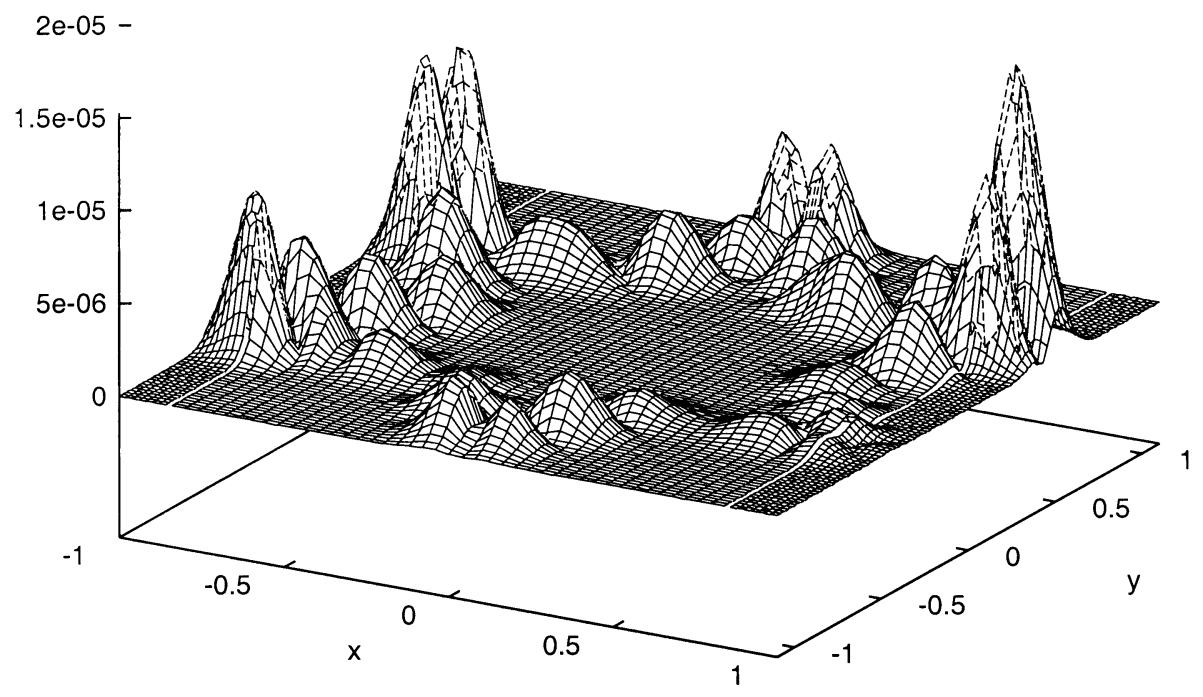

Fig. 6. The output of the RBF network for the domain of problem 2.

is a very desirable feature that is attributed to the MLP interpolation capability (the RBF contributes only around the boundary) and constitutes one of the assets of the proposed method.

Problem 3: We have solved the previous nonlinear PDE considering the cardioid domain displayed in Fig. 7. We have used $M=100$ boundary points and $K=500$ grid points displayed in Fig. 6. An MLP with 20 hidden units was used. The accuracy of the obtained solution (with Neumann BCs) at a dense grid of interpolation points is shown in Fig. 8. The results are similar for the case of Dirichlet BCs.

\section{B. Three Dimensional Problem}

Problem 4: We considered the problem

$$
\begin{aligned}
\nabla^{2} \Psi(x, y, z)= & \Psi^{2}(x, y, z)+e^{x} y^{2}+z^{2} \sin (y) \\
& -\left(e^{x} y^{2}+\left(z^{2}-2\right) \sin (y)\right)^{2} .
\end{aligned}
$$

The domain is most conveniently described in spherical coordinates $(r, \phi, \theta)$ as: $r \in[0.5,1], \phi \in[0, \pi / 2], \theta \in[0, \pi / 2]$.
The problem, however, is solved using Cartesian coordinates $(x, y, z)$.

The analytical solution is $\Psi_{a}(x, y, z)=e^{x} y^{2}+\left(z^{2}-\right.$ 2) $\sin (y)$. We considered $M=176$ boundary points and $K=729$ grid points and solve the nonlinear equation with both Dirichlet and Neumann BCs. The obtained solutions using an MLP with 40 hidden units are accurate with absolute error value less than $10^{-5}$.

\section{Convergence and Stability Issues}

In order to investigate the convergence properties of the method, we conducted several numerical experiments using the nonlinear example of problem 2 with Dirichlet BCs. Specifically we calculated the approximation error in the max norm for several choices of the number of the hidden MLP units.

This is plotted in Fig. 9. Notice that it is very similar to earlier findings [1], where in addition a comparison to the performance of the finite elements method is provided. We see that the accuracy can be controlled efficiently by varying the number of the 


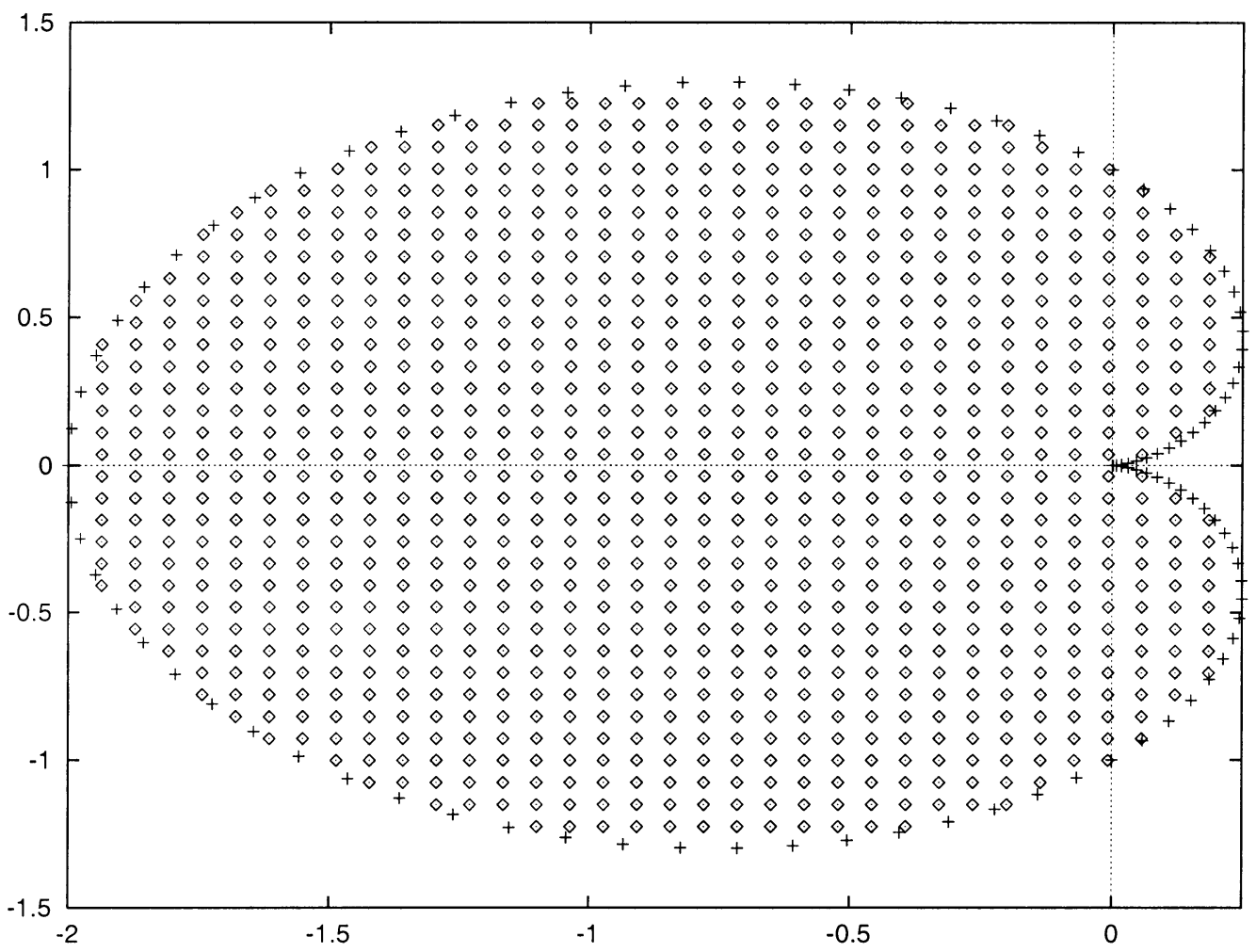

Fig. 7. The domain and the boundary points for problem 3. Boundary points are shown as crosses.

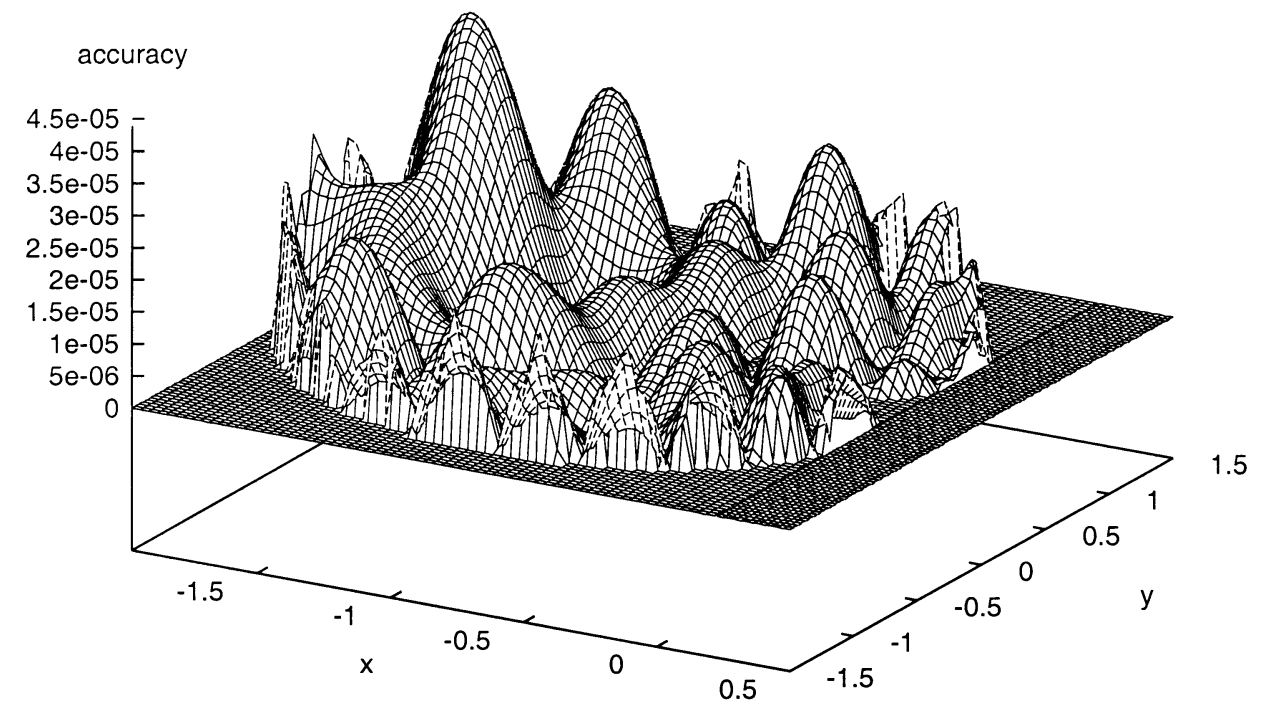

Fig. 8. Accuracy of obtained solution for problem 3 with Neumann BCs.

hidden units. We also investigated how the solution is affected by considering different boundary point sets, while keeping all other computational parameters unaltered. We conducted our experiments again with problem 2 as above.

Let us denote by $M$ the total number of the points on the boundary. The star shaped boundary has 12 vertices (and 12 sides). On each side equal number of points were considered and care has been taken that the star vertices are always included. We also experimented with various distributions, for instance uniform and several sinusoidal forms. An extreme case is $M=12$, i.e., only the star vertices are taken as representative boundary points. For $M=12$ to 48 we observed a slight variation among the obtained solutions. For $M=48$ and above the obtained solutions are identical. In Table I we list the approximation error in the max norm for several choices of $M$, using an MLP with 20 hidden units. In addition, we investigated the case where a vertex is intentionally excluded from the set for the extreme case of $M=11$ and also for the case $M=47$. The solution for the former case is eminently different only around the omitted vertex and the pointwise approximation error is plotted in Fig. 10. Notice that the approximation error in the area of the missing vertex is of the order $4 \times 10^{-3}$. For $M=47$ (again 


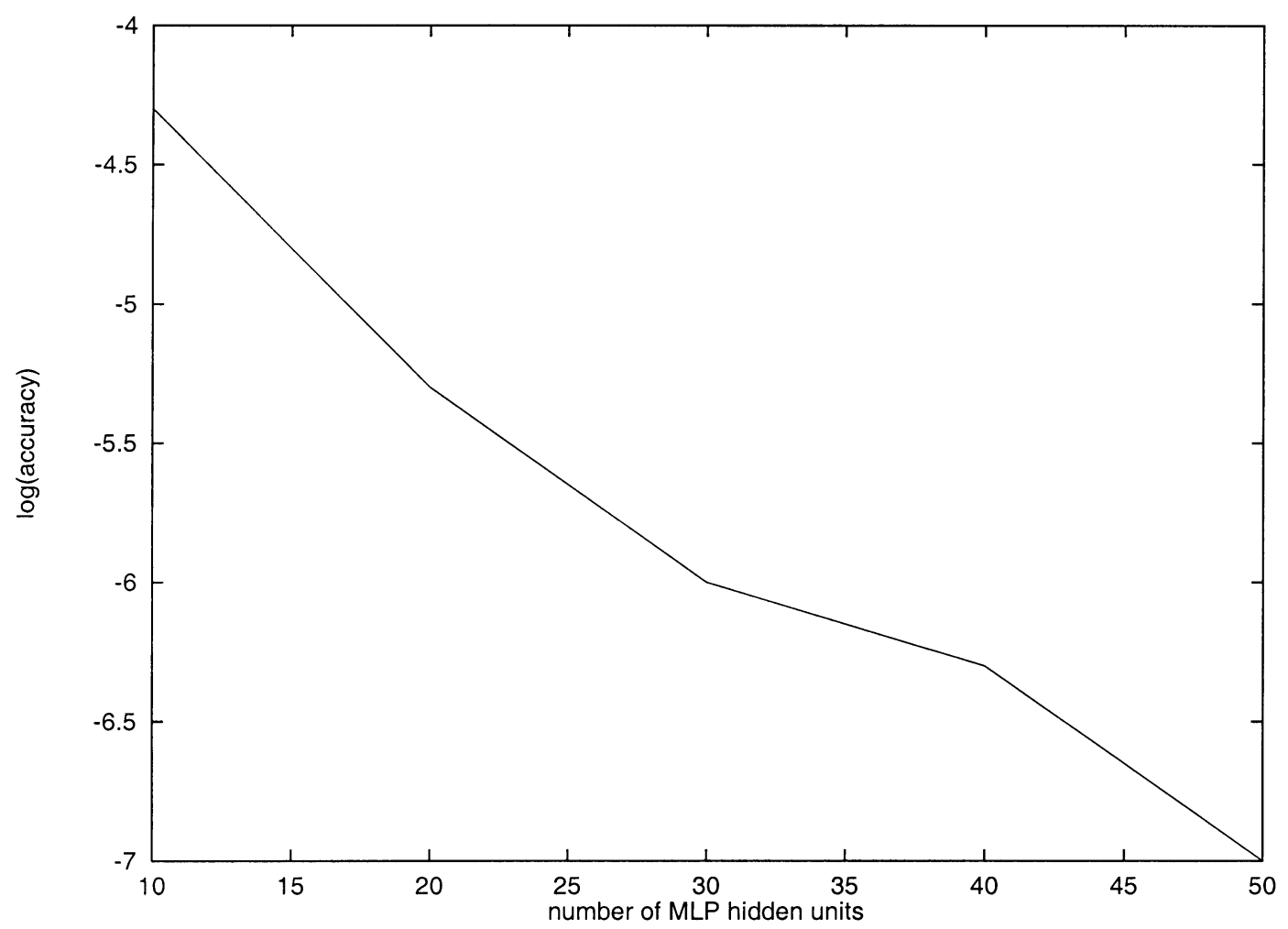

Fig. 9. Plot of the logarithm of the approximation error in the max-norm as a function of the number of MLP hidden units.

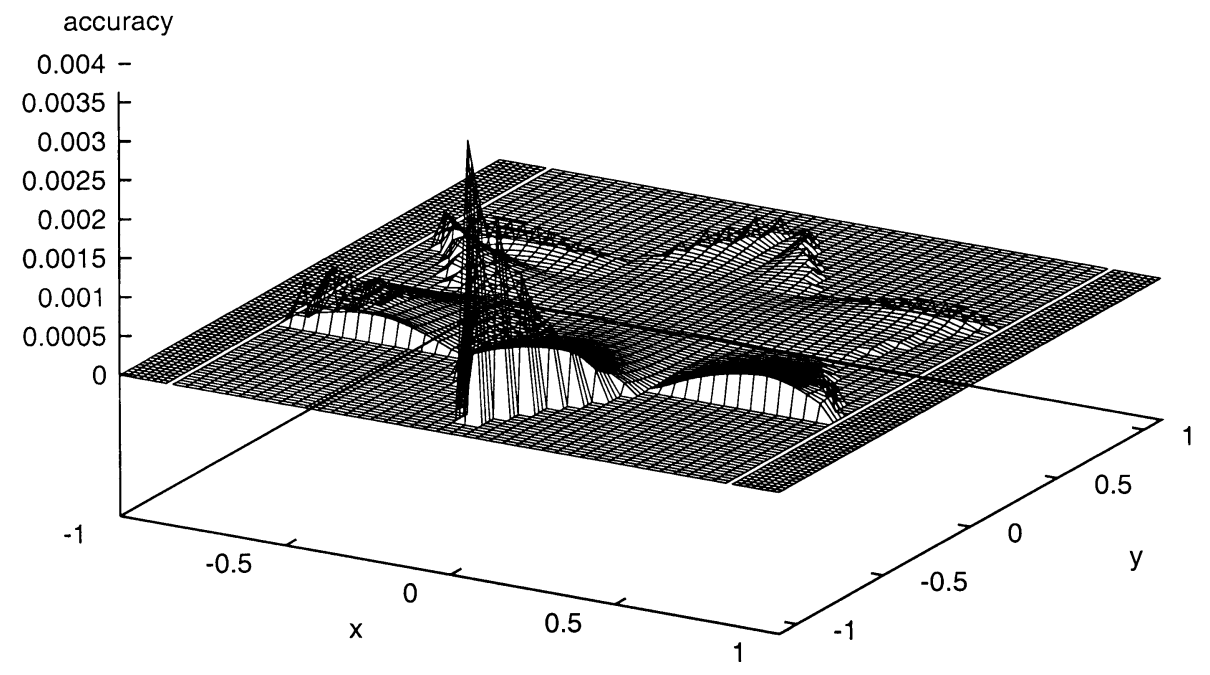

Fig. 10. Accuracy of the obtained solution for problem 2 with Dirichlet BCs when the boundary contains only the star vertices and one of them is missing.

TABLE I

APPROXIMATION ERROR FOR PROBLEM 2 WITH DIRICHLET BCS FOR DIFFERENT CHOICES OF THE BOUNDARY SET

\begin{tabular}{c|c}
\hline Number of Boundary Points & Accuracy \\
\hline 12 & $5 \times 10^{-5}$ \\
\hline 24 & $10^{-5}$ \\
\hline 48 & $5 \times 10^{-6}$ \\
\hline 72 & $5 \times 10^{-6}$ \\
\hline 96 & $5 \times 10^{-6}$ \\
\hline
\end{tabular}

with one vertex ommited) this phenomenon, while still being present, is supressed by two orders of magnitude $\left(5 \times 10^{-5}\right)$.
This is both expected since, the boundary is poorly represented when some vertex is ommited, and at the same time desirable, since it demonstrates that the boundary does indeed, as it should, affect the solution. Hence we conclude that the method yields consistent results and therefore is suitable for application to real problems.

\section{CONCLUSION}

We presented a method capable of solving boundary value problems of the Dirichlet and Neumann types, for boundaries that due to their geometrical complexity can only be described 
via a set of participating points. The method employs the collocation as well as optimization techniques and is based on the synergy of MLP and RBF artificial neural networks. It provides accurate solutions in a closed analytic form that satisfy the BCs at the selected points exactly. The proposed method is quite general and can be used for a wide class of linear and nonlinear PDEs.

Future work will focus on the application of the method to specific problems, containing real objects with arbitrarily complex boundaries. Interesting problems of this kind arise in many scientific fields. Since the method lends itself to parallel processing, we have a strong interest in implementing the method on both, general purpose parallel hardware and on specialized hardware (neuroprocessors). The latter would reveal the full potential of the proposed approach and may lead to the development of specialized machines that will allow the treatment of difficult and computationally demanding problems.

\section{ACKNOWLEDGMENT}

I. E. Lagaris wishes to acknowledge the warm hospitality offered by Prof. Ishii and Prof. Tsoukalas of Purdue University, School of Nuclear Engineering, during his stay at Lafayette, where part of this work was carried out.

\section{REFERENCES}

[1] I. E. Lagaris, A. Likas, and D. I. Fotiadis, "Artificial neural networks for solving ordinary and partial differential equations," IEEE Trans. Neural Networks, vol. 9, pp. 987-1000, 1998.

[2] —_, "Artificial neural network methods in quantum mechanics," Comput. Phys. Commun., vol. 104, pp. 1-14, 1997.

[3] A. Charalambopoulos, G. Dassios, D. I. Fotiadis, and C. Massalas, "Frequency spectrum of the human-neck system," Int. J. Eng. Sci., vol. 35, pp. 753-768, 1997.

[4] D. I. Fotiadis, M. Boekholt, K. Jensen, and W. Richter, "Flow and heat transfer in CVD reactors: Comparison of Raman temperature measurement and finite element method prediction," J. Crystal Growth, vol. 100, pp. 577-599, 1990.

[5] D. G. Papageorgiou, I. N. Demetropoulos, and I. E. Lagaris, "Merlin-3.0, a multidimensional optimization environment," Comput. Phys. Commun., vol. 109, pp. 227-249, 1998.

[6] -, "The Merlin control language for strategic optimization," Comput. Phys. Commun., vol. 109, pp. 250-275, 1998.

[7] K. Hornik, M. Stinchcombe, and H. White, "Multilayer feedforward networks are universal approximators," Neural Networks, vol. 2, pp. 359-366, 1989

[8] M. Leshno, V. Lin, A. Pinkus, and S. Schocken, "Multilayer feedforward networks with nonpolynomial activation function can approximate any function," Neural Networks, vol. 6, pp. 861-867, 1993.
[9] D. Kincaid and W. Cheney, Numerical Analysis. Pacific Grove, CA: Brooks/Cole, 1991.

[10] A. Likas, D. A. Karras, and I. E. Lagaris, "Neural-network training and simulation using a multidimensional optimization system," Int. J. Comput. Math., vol. 67, pp. 33-46, 1998.

[11] R. Fletcher, Practical Methods of Optimization, 2nd ed. New York: Wiley, 1987.

[12] V. Simoncini and E. Gallopoulos, "An iterative method for nonsymmetric systems with multiple right-hand sides," SIAM J. Sci. Comput., vol. 16, pp. 917-933, 1998 .

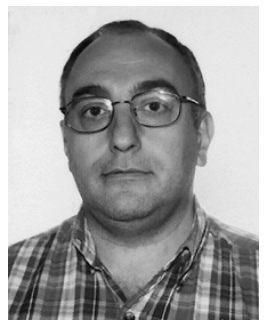

Isaac Elias Lagaris received the B.Sc. degree in physics from the University of Ioannina, Greece, in 1975. He received the M.Sc. degree in 1977 and the Ph.D. degree in 1981, both from the Physics Department of the University of Illinois, Urbana-Champaign.

He was a Lecturer in the Physics Department of the University of Ioannina and since 1994 he has been an Associate Professor in the Department of Computer Science. His research interests include modeling and simulation of classical and quantum systems, highperformance computing, optimization, and neural networks.

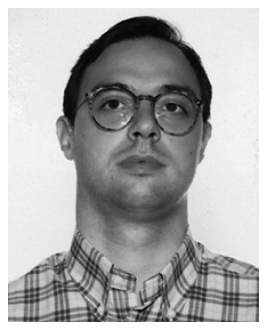

Aristidis C. Likas (S'91-M'96) received the Diploma degree in electrical engineering in 1990 and the Ph.D. degree in electrical and computer engineering in 1994, both from the National Technical University of Athens. Since 1996, he has been with the Department of Computer Science, University of Ioannina, Greece,

$\mathrm{He}$ is currently a Lecturer with the Department of Computer Science, University of Ioannina. His research interests include neural networks, pattern recognition, machine learning, and optimization.

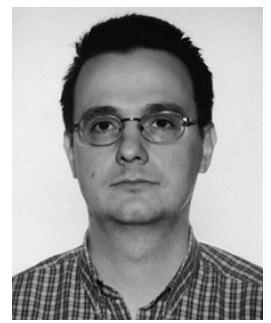

Dimitrios G. Papageorgiou received the B.Sc. degree in physics and the Ph.D. degree from the Department of Chemistry, University of Ioannina, Greece, in in 1989 and 1997, respectively.

He worked for three years at the Network Operations Center of the University of Ioannina and currently is a Research Associate in the department of Materials science. His research interests include optimization methods, computational physical chemistry, simulation of solids and surfaces, neural networks, and high-performance computing. 